\title{
Brightness temperature constraints from interferometric visibilities
}

\author{
Andrei Lobanov ${ }^{1,2}$ \\ ${ }^{1}$ Max-Planck-Institut für Radioastronomie, Auf dem Hügel 69, 53121 Bonn, Germany \\ e-mail: alobanov@mpifr-bonn.mpg.de \\ 2 Institut für Experimentalphysik, Universität Hamburg, Luruper Chaussee 149, 22761 Hamburg, Germany
}

Received 30 September 2014 / Accepted 4 December 2014

\section{ABSTRACT}

Context. The brightness temperature is an effective parameter that describes the physical properties of emitting material in astrophysical objects. It is commonly determined by imaging and modeling the structure of the emitting region and estimating its flux density and angular size.

Aims. Reliable approaches for visibility-based estimates of brightness temperature are needed for interferometric experiments in which poor coverage of spatial frequencies prevents successful imaging of the source structure, for example, in interferometric measurements made at millimeter wavelengths or with orbiting antennas.

Methods. Such approaches can be developed by analyzing the relations between brightness temperature and visibility amplitude and its rms error.

Results. A method is introduced for directly calculating the lower and upper limits of the brightness temperature from visibility measurements. The visibility-based brightness temperature estimates are shown to agree well with the image-based estimates obtained in the $2 \mathrm{~cm}$ MOJAVE survey and the $3 \mathrm{~mm}$ CMVA survey, with good agreement achieved for interferometric measurements at spatial frequencies exceeding $\approx 2 \times 10^{8}$.

Conclusions. The method provides an essential tool for constraining brightness temperature in all interferometric experiments with poor imaging capability.

Key words. galaxies: jets - techniques: interferometric - methods: analytical

\section{Introduction}

Interferometric measurements offer a powerful tool for probing the finest structures of emitting objects by extending the effective instrumental diameter to the maximum distance (baseline length) between individual elements of an interferometer. However, for interferometric measurements made at extreme baseline lengths, imaging the structure of the target object becomes increasingly limited, owing to incomplete sampling of the Fourier plane. This is often the case in radio interferometric measurements made with very long baseline interferometry (VLBI) at millimeter wavelengths (cf. Doeleman et al. 2012) and with space-ground interferometers such as VSOP (Horiuchi et al. 2004) or RadioAstron (Kardashev et al. 2013). In these situations, more basic measurements of flux density, $S_{v}$, and emitting area, $\Omega$, of the structure can still be obtained (i.e., from model fitting of the visibility distribution) and can be combined to yield a brightness temperature estimate. The latter can then be used as a generic indicator of the physical conditions of the emitting material (cf. Lobanov et al. 2000; Kovalev et al. 2005; Homan et al. 2006; Lee et al. 2008).

For the black-body spectrum in the Rayleigh-Jeans limit ( $h v \ll k T)$, the brightness, $I_{v}$, is approximated by $I_{v}=$ $2 v^{2} k T / c^{2}$, and the respective brightness temperature is $T_{\mathrm{b}}=$ $I_{\nu} c^{2} /\left(2 k v^{2}\right)$, where $h$ are $k$ are the Planck and Boltzmann constants, respectively, and $c$ is the speed of light. In terms of the measured quantities, $S_{v}$ and $\Omega$, the resulting brightness is $I_{v}=S_{v} / \Omega=S_{v} /\left[2 \pi\left(1-\cos \rho_{\mathrm{d}}\right)\right]$, if the emitting region is a uniformly bright circle of angular radius $\rho_{\mathrm{d}}$. For small $\rho_{\mathrm{d}}$, the term $1-\cos \rho_{\mathrm{d}}$ is approximated by $\rho_{\mathrm{d}}^{2} / 2$, which yields $I_{v} \approx S_{v} /\left(\pi \rho_{\mathrm{d}}^{2}\right)$.
If the emitting region is unresolved, $\rho_{\mathrm{d}}$ can be constrained by the resolution limit, $\theta_{\mathrm{lim}}$, of the measurement, providing lower limits on the brightness, $I_{v} \geq 4 S_{v} /\left(\pi \theta_{\mathrm{lim}}^{2}\right)$, and brightness temperature, $T_{\mathrm{b}} \geq 2 S_{\nu} c^{2} /\left(\pi k v^{2} \theta_{\mathrm{lim}}^{2}\right)$.

In absence of information about the actual brightness distribution of emission in a compact, marginally resolved region, it is often assumed that it can be represented satisfactorily by a two-dimensional Gaussian distribution described by a flux density $S_{\mathrm{g}}$ and respective major and minor axes $\theta_{\text {maj }}$ and $\theta_{\text {min }}$. This translates into $I_{v}=(4 \ln 2 / \pi) S_{\mathrm{g}} /\left(\theta_{\text {maj }} \theta_{\text {min }}\right)$ and $T_{\mathrm{b}}=[2 \ln 2 /(\pi k)] S_{\mathrm{g}} c^{2} /\left(v^{2} \theta_{\text {maj }} \theta_{\text {min }}\right)$. These expressions are used for the bulk of brightness temperature estimates based on decomposition of the observed structure into one or more twodimensional Gaussian features (Gaussian components). Several other analytical patterns of brightness distribution patterns have been employed to analyze different astrophysical objects (cf. Berger 2003) such as resolved stars (Dyck et al. 1998; Ohnaka et al. 2013), young supernovae (Marcaide et al. 2009), recurrent novae (Chesneau et al. 2007), protoplanetary disks (Malbet et al. 2005), or active galaxies (Weigelt et al. 2012). In all of these cases, successful fitting of a given brightness distribution pattern to visibility data is a strong prerequisite for recovering structural and physical information about the target object.

However, the most extreme cases of interferometric observations, such as millimeter and space VLBI measurements, often do not provide enough data to warrant reliable model fitting owing to lack of short-baseline measurements and the complexity of the fine structure in most of the targets. These observations require a different approach for estimating the brightness temperature. In this paper, such an approach is proposed, based on 
individual visibility measurements and their errors (which can be reliably estimated in most of the measurements). The methodology of this approach is described in Sect. 2 and is tested with the visibility data from two $\mathrm{VLBA}^{1}$ observations of the prominent compact radio sources 3C 345 and NGC 1052. Applications of the visibility-based $T_{\mathrm{b}}$ estimates are discussed in Sect. 3 and are compared with the results from the $3 \mathrm{~mm} \mathrm{CMVA}^{2}$ and $2 \mathrm{~cm}$ MOJAVE ${ }^{3}$ surveys and in connection with an analysis of space VLBI and millimeter VLBI surveys of compact radio sources. Additional potential applications of the method to other types of astrophysical targets are also discussed, and expressions for brightness temperature limits for several specific brightness distribution patterns are presented in the appendix.

\section{Brightness temperature limits from interferometric visibilities}

We consider an emitting region with a brightness distribution, $I_{r}$, observed instantaneously at a wavelength, $\lambda$, by an interferometer consisting of two receiving elements (telescopes) separated by a baseline distance, $B$. This observation corresponds to measuring the Fourier transform of $I_{r}$ at a single spatial (Fourier) frequency $q=B / \lambda$ (also called $u v$ distance or $u v$ radius). It yields an interferometric visibility, $V=V_{q} \mathrm{e}^{-i \phi_{q}}$, described by its amplitude $V_{q}$ and phase $\phi_{q}$, and their respective errors $\sigma_{q}$ and $\sigma_{\phi}$. Generally speaking, $V_{q}$ depends on the shape and angular extent of the brightness distribution, and $\phi_{q}=\phi_{\mathrm{p}}+\phi_{\mathrm{o}}$ is a function of its position and geometry. The position-dependent term of the phase, $\phi_{\mathrm{p}}$, is relative and can always be zeroed by an appropriate shift applied to the visibility (which is analogous to re-pointing the interferometer). The geometry-dependent term of the phase, $\phi_{\mathrm{o}}$, depends on the structure of the brightness distribution and its orientation with respect to the projection of the interferometric baseline on the picture plane. For a circularly symmetric or axially symmetric brightness distribution, $\phi_{\mathrm{o}} \equiv 0$, independently of the baseline orientation.

\subsection{Minimum brightness temperature}

Without a priori information about the specific structural shape of $I_{r}$, the symmetry assumption can be employed and the angular extent of the emission can be estimated from $V_{q}$ alone. This assumption is routinely used for size and brightness temperature estimates made from interferometric data (cf. Lobanov et al. 2000; Kovalev et al. 2005; Lee et al. 2008).

Such estimates require knowledge of the zero-spacing visibility, $V_{0}$, and rely upon assumption of a specific symmetric template for $I_{r}$. For instance, for a circular Gaussian distribution, the respective expression for $V_{q}$ is

$$
V_{q}=V_{0} \exp \left(-\frac{\pi^{2} \theta_{r}^{2} q^{2}}{4 \ln 2}\right)
$$

and it can be used for obtaining an estimate of the size, $\theta_{r}$, of the emitting region:

$\theta_{r}=\frac{2 \sqrt{\ln 2}}{\pi} \frac{\lambda}{B} \sqrt{\ln \left(V_{0} / V_{q}\right)}$.

\footnotetext{
1 Very Long Baseline Array of National Radio Astronomy Observatory, Socorro NM, USA; http: //www . nrao. edu

2 Coordinated Millimeter VLBI Array, currently succeeded by the Global Millimeter VLBI Array; http://www3.mpifr-bonn.mpg. de/div/vlbi/globalmm/

3 http://Www . phyiscs . purdue.edu/astro/MOJAVE/
}

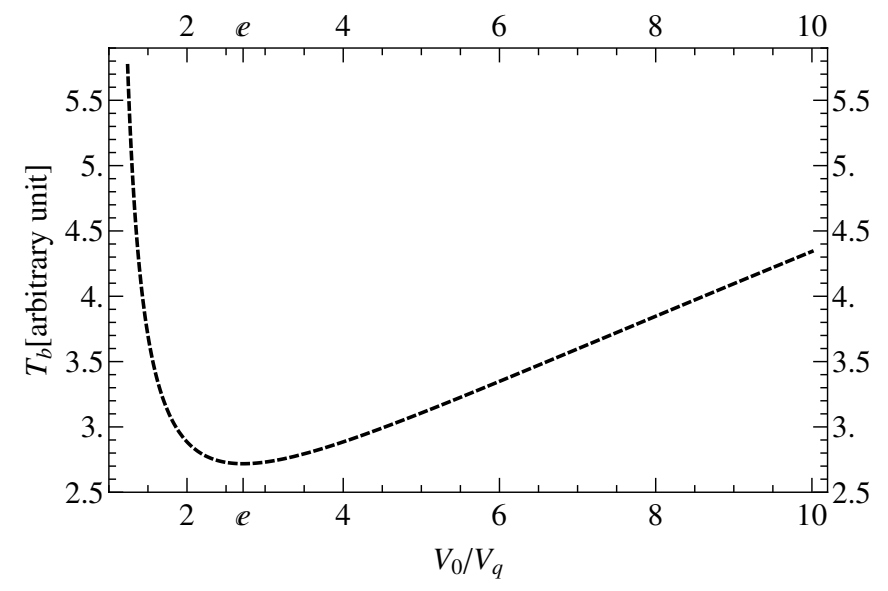

Fig. 1. Brightness temperature in relative units as a function of the ratio $V_{0} / V_{q}$ between the zero-spacing flux density and visibility flux density measured at a given spatial frequency $q$, assuming that the brightness distribution is Gaussian. The lowest value of the brightness temperature is realized with the ratio $V_{0} / V_{q}=e$.

With this expression, the brightness temperature can be estimated from

$T_{\mathrm{b}}=\frac{\pi}{2 k} \frac{B^{2} V_{0}}{\ln \left(V_{0} / V_{q}\right)}$.

Appendix A lists the respective expressions derived for several other patterns of brightness distributions commonly used in the analysis of astronomical data.

Equation (2) provides the lowest value of $T_{\mathrm{b}}$ for $V_{0}=e V_{q}$ (see Fig. 1), which yields an estimate of the minimum brightness temperature supported by the measured visibility amplitude $V_{q}$ :

$T_{\mathrm{b}, \min }=\frac{\pi e}{2 k} B^{2} V_{q} \approx 3.09\left(\frac{B}{\mathrm{~km}}\right)^{2}\left(\frac{V_{q}}{\mathrm{mJy}}\right)[\mathrm{K}]$.

This expression describes the absolute minimum of the brightness temperature that can be obtained from the measured visibility amplitude $V_{q}$ under the assumption that the brightness distribution is well approximated by a circular Gaussian. The lowest brightness temperature is realized for any visibility distribution that has an inflection point. The respective expressions of $T_{\mathrm{b} \text {,min }}$ for other patterns of brightness distributions are listed in Appendix A.

\subsection{Maximum measurable brightness temperature}

The expression for $T_{\mathrm{b} \text {,min }}$ given by Eq. (3) is independent of $V_{0}$, while estimating the maximum brightness temperature will necessarily require knowledge, or at least a reasonable assumption, of $V_{0}$. With the latter, it should be kept in mind that if only a limit on $V_{0}$ can be assumed, the nature of the resulting estimate of $T_{\mathrm{b}}$ depends on the ratio of $V_{0} / V_{q}$. The maximum $T_{\mathrm{b}}$ can only be derived from upper limits on $V_{0}$ for $V_{0}>e V_{q}$ and from lower limits on $V_{0}$ for $V_{0}<e V_{q}$. In the opposite cases, the combination of $V_{0}$ and $V_{q}$ yields an estimate of the minimum brightness temperature.

The zero-spacing visibility $V_{0}$ is often approximated by the total flux density, $S_{\text {tot }}$, measured at a single receiving element. Generally, this is a poor approximation because $S_{\text {tot }}$ contains contributions from all angular scales. A better constraint on $V_{0}$ is provided by the flux density of a respective Gaussian component if the data warrant reliable Gaussian decomposition. For 
extremely poor coverages of the Fourier domain, this is not the case, and no satisfactory estimate of $V_{0}$ can be made.

In this situation, a lower limit of $V_{0}=V_{q}+\sigma_{q}$ can be adopted. This limit effectively corresponds to requiring that $V_{q}$ probes a structural detail that is marginally resolved (we recall that $V_{q} \equiv$ const. results from the Fourier transform of a point source). This assumption is well justified for visibility measurements made at sufficiently long baselines, where the visibility amplitude is dominated by the most compact structure observed in the target object. It can furthermore be verified through the observed absence of amplitude beating at slightly shorter baselines (with the latter reflecting the presence of multiple compact emitting regions in the object). Examples of visibility (baseline) ranges dominated by contributions from the most compact structures are shown in Fig. 2 for two radio sources (NGC 1052 and $3 \mathrm{C} 345)$ that represent the typical cases of a compact radio source with and without a strong contribution from extended emission.

The requirement of marginal resolution of the observed structure implies that its size should be larger than

$\theta_{\lim }=\frac{2 \sqrt{\ln 2}}{\pi} \frac{\lambda}{B} \sqrt{\ln \frac{V_{q}+\sigma_{q}}{V_{q}}}$.

Correspondingly, the brightness temperature of this feature should not exceed the limit of

$$
\begin{aligned}
T_{\mathrm{b}, \lim } & =\frac{\pi B^{2}\left(V_{q}+\sigma_{q}\right)}{2 k}\left[\ln \frac{V_{q}+\sigma_{q}}{V_{q}}\right]^{-1} \\
& =1.14\left(\frac{V_{q}+\sigma_{q}}{\mathrm{mJy}}\right)\left(\frac{B}{\mathrm{~km}}\right)^{2}\left(\ln \frac{V_{q}+\sigma_{q}}{V_{q}}\right)^{-1}[\mathrm{~K}] .
\end{aligned}
$$

For visibilities with a signal-to-noise ratio $V_{q} / \sigma_{q}>e-1$, the estimate of $T_{\mathrm{b}, \text { lim }}$ provides the highest brightness temperature that can be obtained from the measured visibility amplitude and its error while requiring that the respective brightness distribution is a) circularly Gaussian; and b) marginally resolved by the measured visibility. Expressions for $T_{\mathrm{b} \text {,lim }}$ derived for several other brightness distribution patterns are given in Appendix A.

As measurements of $V_{q}$ and $\sigma_{q}$ are made over extended time intervals, they correspond to averaging the visibility function over finite ranges of spatial frequencies $(\Delta q, \Delta \psi)$, with $\psi$ describing the positional angle of $V_{q}$ in the Fourier plane. In this case, the estimate provided by Eq. (5) holds for as long as the condition $V_{\mathrm{q}, \psi} \approx$ const. is satisfied over the given measurement interval $[\Delta q, \Delta \psi]$. This condition effectively requires that the measured $V_{q}$ is dominated by a contribution from a single emitting region that is marginally resolved at the spatial frequency $q$.

Equations (3) and (5) provide a robust bracketing for the brightness temperature obtained from interferometric measurements that have a limited sampling of the visibility distribution of the target. This can be demonstrated by applying these equations to every visibility of the VLBI datasets used in the examples shown in Fig. 2. Results of this application are presented in Fig. 3, where the visibility-based estimates of $T_{\mathrm{b} \text {,min }}$ and $T_{\mathrm{b}, \mathrm{lim}}$ are plotted against the spatial frequency ( $u v$ radius) of the respective visibilities. These estimates can be compared with the brightness temperature, $T_{\mathrm{b} \text {,mod }}$ estimated from the model fit parameters of the "core" component. This comparison indicates that at $u v$ radii $\gtrsim 150 \mathrm{M} \lambda$, the interval $\left[T_{\mathrm{b}, \min }, T_{\mathrm{b}, \mathrm{lim}}\right]$ represents a reasonably good bracketing for the expected maximum brightness temperature. This substantially exceeds the conservative expectations for the $u v$ ranges (indicated by braces in
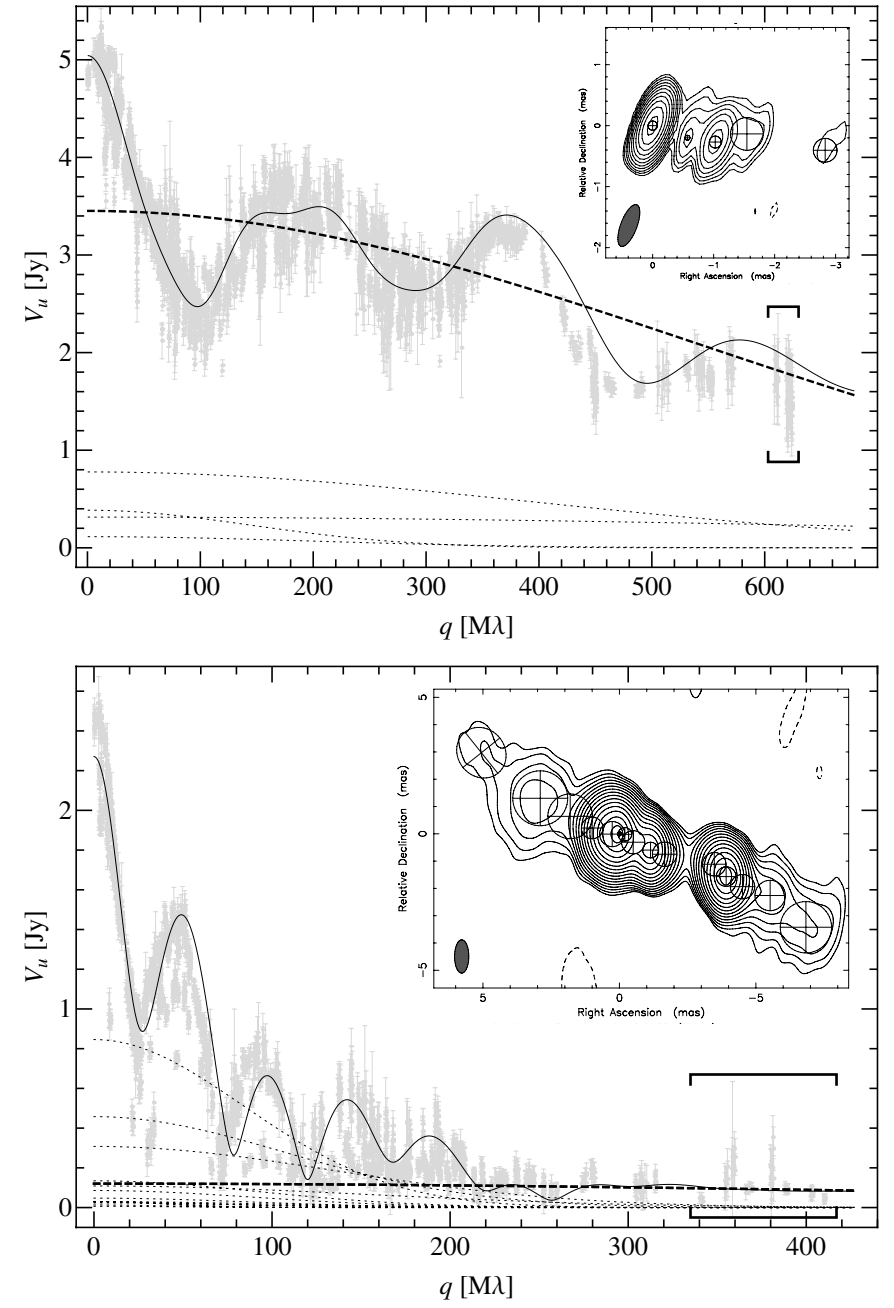

Fig. 2. Comparison of the visibility amplitude distribution and Gaussian modelfit representation of the compact structure in a compact structuredominated object (top, a $22 \mathrm{GHz}$ VLBA observation of the quasar 3C 345 made on 23/08/1999) and an extended structure-dominated object (bottom, a $15 \mathrm{GHz}$ VLBA observation of the radio galaxy NGC 1052 from MOJAVE Survey, made on 16/12/1995; Kellermann et al. 2004). The visibility amplitude distributions are projected onto a PA of $-104^{\circ}(3 \mathrm{C} 345)$ and $-110^{\circ}$ (NGC 1052) for illustration purposes, enabling a representation by a single, composite modelfit rather than by fits to individual baselines. The insets show Gaussian modelfit images of the source structure, with component locations and sizes indicated. The resulting fits of the visibility amplitudes are shown by the solid curves. The dotted curves show visibility representations of the individual modelfit components. The thick dashed lines correspond to the respective visibility responses from the most compact "core" component that has the highest brightness temperature. Braces indicate the spatial frequency ( $u v$ distance) ranges in which the contribution from the core component dominates the measured visibility amplitude distribution (the $u v$ ranges are measured in units of $\mathrm{M} \lambda$, where $\lambda$ is the observing wavelength). These spatial frequency ranges are best suited to directly estimate the brightness temperature from the visibility measurements.

Fig. 3) suitable for estimating the brightness temperature. Within these ranges, the average $T_{\mathrm{b}, \mathrm{lim}}$ is only marginally (factors of 1.5 and 2.2) higher than the $T_{\mathrm{b} \text {,mod }}$ obtained from modelfits. Hence, $T_{\mathrm{b}, \mathrm{lim}}$ estimates made at long baselines can constrain the maximum brightness temperature well in both core-dominated and jet-dominated compact radio sources. 

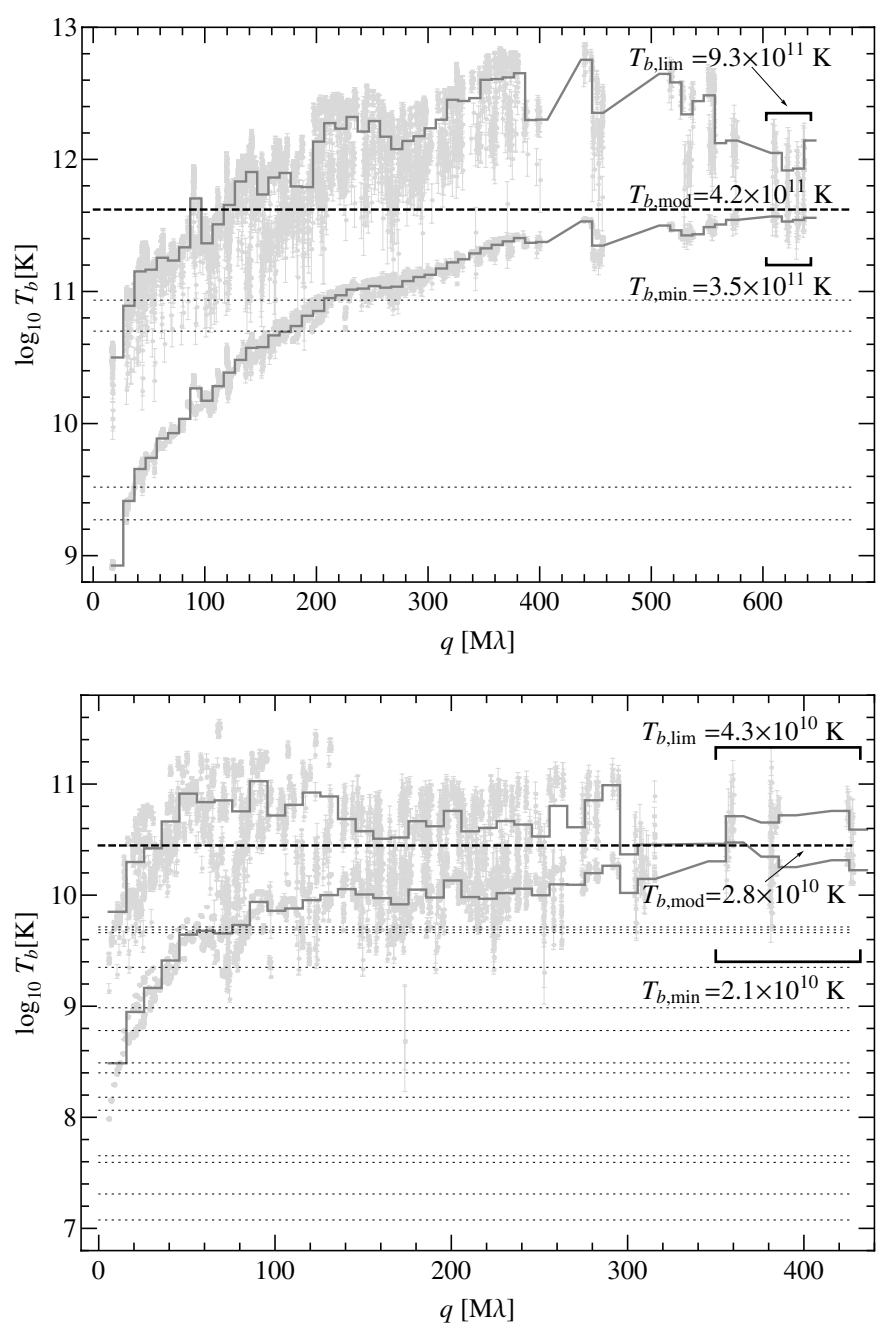

Fig. 3. Limiting, $T_{\mathrm{b}, \lim }$ (top row of datapoints) and minimum, $T_{\mathrm{b}, \min }$ (bottom row of datapoints), brightness temperature estimated directly from visibility data for 3C 345 (top panel) and NGC 1052 (bottom panel), plotted against the spatial frequency $q$ ( $u v$ radius). The estimated values are compared with the brightness temperatures of the Gaussian model fit components (dotted lines for the jet components, thick dashed line for the core component) used to describe the source structure as shown in Fig. 2. Running stairs show the respective rows of the brightness temperature averaged within radial bins of $10 \mathrm{M} \lambda$ in extent. Both the original and the averaged rows of the brightness temperature indicate that at $q \gtrsim 150 \mathrm{M} \lambda$, the interval $\left[T_{\mathrm{b}, \min }, T_{\mathrm{b}, \mathrm{im}}\right]$ provides a good bracketing for the maximum brightness temperature in each of the two objects. Braces indicate the conservative ranges of spatial frequency $q$ identified as ranges dominated by the most compact part of the source structure. Averages of $T_{\mathrm{b}, \min }$ and $T_{\mathrm{b}, \text { lim }}$ made over these ranges constrain the respective $T_{\mathrm{b}, \text { mod }}$ estimates well.

\subsection{Corrections for elongation of the emitting region}

The estimates of $T_{\mathrm{b}, \min }$ and $T_{\mathrm{b}, \mathrm{lim}}$ can furthermore be refined if the potential elongation of the emitting region is considered. If visibility measurements are made over a narrow range of position angle $\psi$ in the Fourier plane, this elongation may bias individual estimates of the brightness temperature and introduce scatter in the statistics obtained from object samples. Gaussian modeling of fine structure in the objects from the MOJAVE sample (Kovalev et al. 2005) indicates that the core components are well described by elliptical Gaussian patterns, with an average elongation (minor to major axis ratio, $\epsilon=\theta_{\min } / \theta_{\text {maj }}$ ) of $0.4 \pm 0.2$.

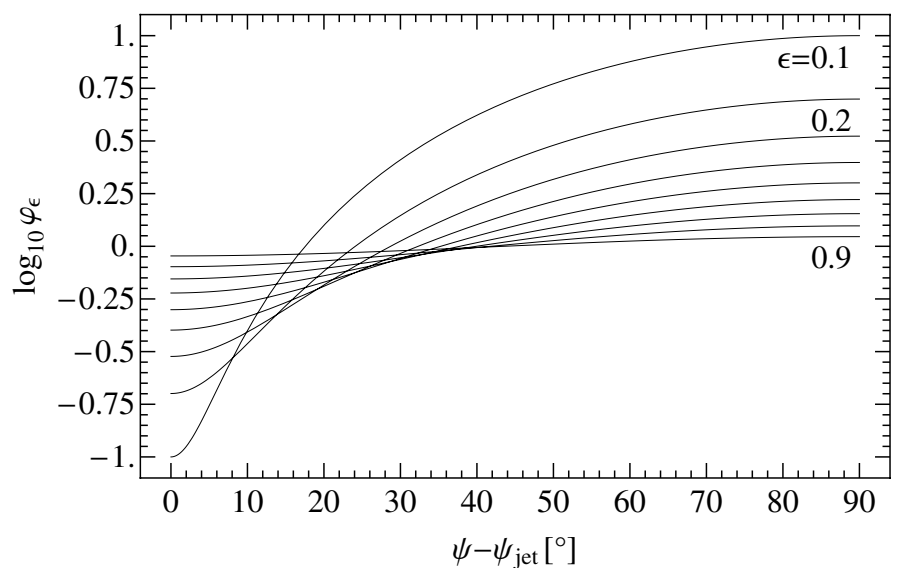

Fig. 4. Brightness temperature correction to account for the elongation of the emitting region. The elongated region is described by an elliptical Gaussian with an axial ratio $\epsilon$. The corrections are plotted against the difference between the position angle of a visibility measurement and the position angle of the major axis of the Gaussian. Different curves correspond to the corrections for values of $\epsilon$ taken in steps of 0.1 between $\epsilon=0.1$ and $\epsilon=0.9$.

For visibility measurements made at a random position angle $\psi$ (hence oriented randomly with respect to the source elongation), the resulting scatter of $T_{\mathrm{b}}$ estimates can approach one order of magnitude. This adverse effect of the source elongation can be taken into account in objects with a known elongation and position angle of the compact structure.

The compact core region can be approximated by an elliptical Gaussian with an axial ratio, $\epsilon$, and a position angle of the major axis, $\psi_{\text {jet }}$. The angular size estimate, $\theta_{r}$, obtained under the assumption of a circular Gaussian brightness distribution (e.g., with Eq. (1)) can then be related to the major and minor axes of the elliptical Gaussian,

$$
\begin{aligned}
& \theta_{\text {maj }}=\theta_{r} / \sqrt{\sin ^{2} \zeta+\epsilon^{2} \cos ^{2} \zeta} \\
& \theta_{\text {min }}=\theta_{r} / \sqrt{\cos ^{2} \zeta+\epsilon^{-2} \sin ^{2} \zeta} \equiv \theta_{\text {maj }} \epsilon,
\end{aligned}
$$

where $\zeta=\psi-\psi_{\text {jet }}$ describes the difference between the visibility position angle and that of the major axis of the elliptical Gaussian component (hence $\theta_{r}=\theta_{\text {min }}$ for $\zeta=0^{\circ}$ and $\theta_{r}=\theta_{\text {maj }}$ for $\zeta=90^{\circ}$ ). For the brightness temperature estimates, this results in a multiplicative correction factor

$\varphi_{\epsilon}=\theta_{r}^{2} /\left(\theta_{\text {maj }} \theta_{\text {min }}\right)=\epsilon \cos ^{2} \zeta+\epsilon^{-1} \sin ^{2} \zeta$

that should be applied to $T_{\mathrm{b} \text {,min }}$ and $T_{\mathrm{b} \text {,lim }}$ given by Eqs. (3) and (5). The magnitude of this correction is on the order of $1 / \epsilon^{2}$ over the full range of values of $\zeta$ (see Fig. 4). Applying this correction may be particularly useful for analysis of space VLBI measurements made with RadioAstron at baselines in excess of ten Earth diameters (hence falling within a range of $\Delta \psi \lesssim 6^{\circ}$ ). Other potential applications include snapshot VLBI measurements made at millimeter wavelengths (e.g., Doeleman et al. 2012; Petrov et al. 2012; Lee et al. 2013).

The effect of correcting for the core elongation is illustrated in Fig. 5, where the correcting factor $\varphi_{\epsilon}$ is applied to brightness temperature estimates made from the visibility data on 3 C 345 presented in Figs. 2,3. The corrections are derived for an axial ratio $\epsilon=0.5$ (weighted average of the model-fitting results 


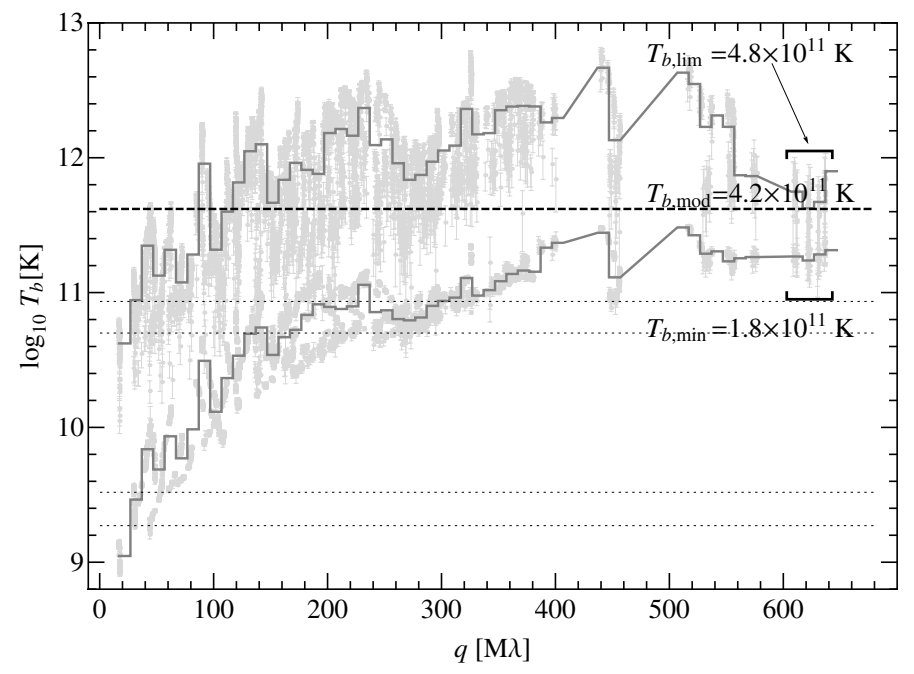

Fig. 5. Limiting, $T_{\mathrm{b}, \lim }$ (top row of datapoints) and minimum, $T_{\mathrm{b}, \min }$ (bottom row of datapoints), brightness temperature estimated directly from visibility data for 3C 345 and corrected for an assumed ellipticity of the emitting region, with $\epsilon=0.5$ (based on MOJAVE model fitting reported in Kovalev et al. 2005). and $\psi_{\text {jet }}=-104^{\circ}$. The limiting brightness temperature of $4.8 \times 10^{11} \mathrm{~K}$, obtained from the visibilities at the longest baselines $(B>600 \mathrm{M} \lambda)$, agrees well with the estimate based on model fitting the source structure.

in Kovalev et al. 2005) and a jet position angle $\psi_{\text {jet }}=-104^{\circ}$ inferred from the source structure shown in Fig. 2. The increased scatter at shorter baselines shows the effect of contributions from larger structures that are incorrectly described by the adopted values of $\phi_{\text {jet }}$ and, in particular, $\epsilon$. However, at the longest baselines, correcting for the core elongation clearly improves the $T_{\mathrm{b}, \mathrm{lim}}$ estimate and brings it well within the errors of the modelfit-based estimate. The same correction applied to NGC 1052 (with $\epsilon=0.4$ and $\psi_{\text {jet }}=-110^{\circ}$ ) results in corrected $T_{\mathrm{b}, \text { min }}=1.1 \times 10^{10} \mathrm{~K}$ and $T_{\mathrm{b}, \mathrm{lim}}=2.4 \times 10^{10} \mathrm{~K}$, with the latter value falling very close to $T_{\mathrm{b} \text {, mod }}=2.8 \times 10^{10} \mathrm{~K}$ estimated from the modelfit. Both these results indicate that $T_{\mathrm{b}, \lim }$ can be improved by correcting for the elongation of the core region.

\section{Discussion}

Applying the visibility-based brightness temperature estimates to VLBI data on 3C 345 and NGC 1052 has demonstrated that the method is reliable in two particular cases. A more extended testing of the method can be performed on a statistical basis by applying it to visibility data from large VLBI survey programs aimed at measuring and analyzing the brightness temperature distribution in samples of compact radio sources. The analysis of fine scale structure in the $15 \mathrm{GHz}\left(\lambda_{\mathrm{obs}}=2 \mathrm{~cm}\right)$ MOJAVE sample (Kovalev et al. 2005) and the results of brightness temperature measurements from the $86 \mathrm{GHz}\left(\lambda_{\mathrm{obs}}=3 \mathrm{~mm}\right)$ CMVA survey Lee et al. (2008) offer statistically suitable samples for such tests. The MOJAVE analysis was based on elliptical Gaussian model fits of the core region. The $86 \mathrm{GHz}$ data were fitted by circular Gaussian components. Resolution criteria were applied to the data from both surveys to constrain the core components with degenerate size parameters (circular diameter or one of the two axes of elliptical Gaussian) obtained from the model fitting.

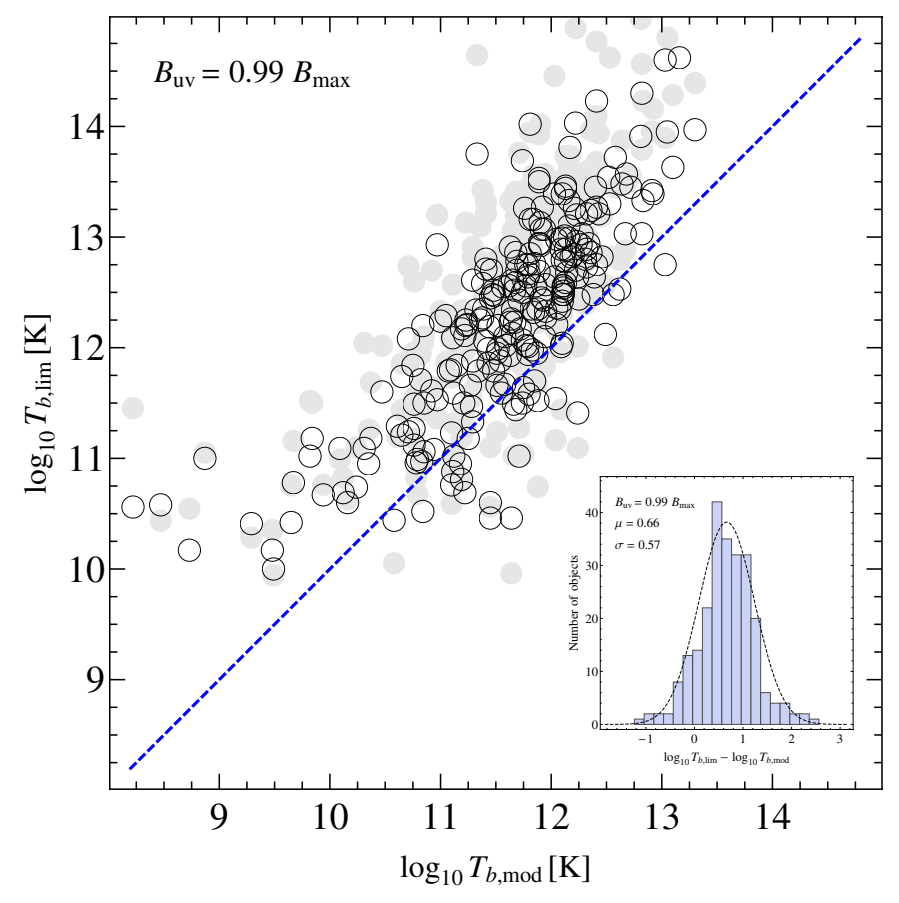

Fig. 6. Comparison of $T_{\mathrm{b}, \mathrm{lim}}$ (circular Gaussian approximation) and $T_{\mathrm{b} \text {,mod }}$ estimates obtained from the MOJAVE data (open circles). Gray circles illustrate the effect of correcting $T_{\mathrm{b}, \mathrm{lim}}$ for the putative elongation of the core region. The dashed line marks the one-to-one correspondence between the two estimates. For each object, the $T_{\mathrm{b}, \mathrm{lim}}$ is estimated from MOJAVE data at $R_{\mathrm{uv}} \geq 0.99 B_{\max }$ to restrict the visibility information to the most compact structures. The resulting $T_{\mathrm{b}, \mathrm{lim}}$ are on average 4.6 times higher than $T_{\mathrm{b} \text {,mod }}$. The residual logarithmic distribution of the $T_{\mathrm{b}, \mathrm{lim}} / T_{\mathrm{b} \text {,mod }}$ ratio (inset) can be approximated by the Gaussian PDF with $\mu=0.66$ and $\sigma=0.57$. At $T_{\mathrm{b}, \text { mod }} \lessgtr 10^{10} \mathrm{~K}$ the $T_{\mathrm{b} \text {,lim }}$ may be biased by large-scale structure contributions in strongly jet-dominated objects.

\subsection{Preparation of the visibility data}

To evaluate the performance of visibility-based brightness temperature limits on a self-consistent statistical basis, visibility data for each source from these two programs (244 objects in the MOJAVE sample and 123 observations of 109 individual objects in the CMVA sample) were radially and azimuthally averaged within a small annulus, $\left(B_{\mathrm{uv}}, B_{\max }\right)$ in the $u v$ plane. The purpose of clipping the data is to reduce the adverse effect of including shorter baselines that are dominated by extended structure. The upper limit of the annulus is determined by the longest baseline $B_{\max }$ found in the data for each individual object. The MOJAVE datasets were clipped at $B_{\mathrm{uv}}=0.99 B_{\max }$ (i.e., in an annulus located within $1 \%$ of $B_{\max }$ ). The CMVA survey data, with substantially fewer visibilities per target object, were clipped at $B_{\mathrm{uv}}=0.9 B_{\max }$. The resulting average $u v$ distances and visibility position angles in the clipped data are $435 \pm 27 \mathrm{M} \lambda$ and $-88^{\circ} \pm 6^{\circ}$ for the MOJAVE data and $2270 \pm 660 \mathrm{M} \lambda$ and $-78^{\circ} \pm 20^{\circ}$ for the CMVA data. In each case, the fraction of visibilities selected is $\sim 1 / N_{\text {bas }}$, where $N_{\text {bas }}$ is the number of baselines in a dataset. Following the conclusions obtained from analyzing the data on 3C 345 and NGC 1052, these $u v$ distances should be sufficiently long to reduce the visibility contamination by large-scale structures to negligible levels. The $T_{\mathrm{b}, \mathrm{lim}}$ estimates were therefore calculated for each source from all of the visibilities clipped and averaged within the respective annuli. The circular Gaussian approximation was used in these calculation. 


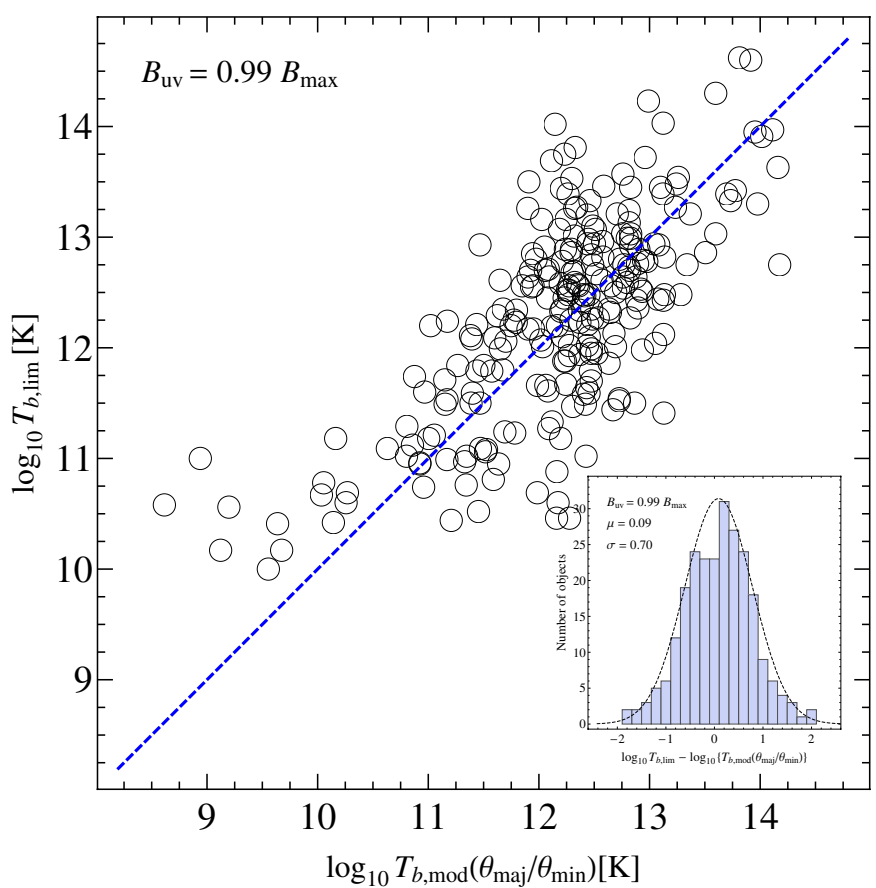

Fig. 7. Correlation between $T_{\mathrm{b}, \mathrm{lim}}$ and $T_{\mathrm{b}, \bmod }\left(\theta_{\mathrm{maj}} / \theta_{\min }\right)$ expressing the brightness temperature obtained assuming that the characteristic size of the brightest region is determined by the jet transverse dimension as given by the minor axis of the elliptical Gaussian fit. The average $T_{\mathrm{b}, \mathrm{lim}}$ is is only $20 \%$ higher than the respective corrected values of $T_{\mathrm{b}, \bmod }$, and the residual logarithmic distribution of the $T_{\mathrm{b}, \mathrm{lim}} / T_{\mathrm{b}, \bmod }$ ratio (inset) can be approximated by the Gaussian PDF with $\mu=0.09$ and $\sigma=0.70$.

\subsection{Results from the MOJAVE data}

For the MOJAVE data, the resulting estimates of $T_{\mathrm{b}, \lim }$ are compared in Fig. 6 with the $T_{\mathrm{b} \text {,mod }}$ estimates obtained in Kovalev et al. (2005) for each source at the same observing epoch. The two estimates agree reasonably well, with $T_{\mathrm{b} \text {,lim }}$ being on average 4.6 times higher than the respective $T_{\mathrm{b} \text {,mod }}$. This is similar to the $T_{\mathrm{b}, \mathrm{lim}} / T_{\mathrm{b} \text {,mod }}$ ratios measured in 3C 345 and NGC 1052. Overall, the $T_{\mathrm{b}, \mathrm{lim}}$ estimates provide a reasonable upper bound on the brightness temperature, with the scatter in the estimates limited to about half a decade over nearly five orders of magnitude in brightness temperature.

Correction for the putative elongation of the core region has been attempted for the MOJAVE data, based on the elliptical model fits and measured position angles of the jet. The elongation was calculated as the ratio of the minor to major axes of the elliptical Gaussian component describing the core. Two options were tried for the jet position angle: a) the position angle of the major axis of the Gaussian component; and b) the average position angle of the jet as reported in Kovalev et al. (2005). The results of applying the elongation correction with the former option are shown in Fig. 6. Neither of the two corrections has improved the correlation for either the average $T_{\mathrm{b}, \mathrm{lim}} / T_{\mathrm{b} \text {,mod }}$ ratio or the spread of the residuals.

This result may be caused by two factors. On the one hand, it may suggest that the elongation of the core region is not accurately reflected in the parameters of the elliptical Gaussian components, for instance, if this region has a specific geometry such as a conically expanding jet (cf. Blandford \& Königl 1979) or a jet pervaded with thread-like instability patterns (cf. Lobanov \& Zensus 2001). On the other hand, the lack of improvement achieved with the elongation correction may indicate

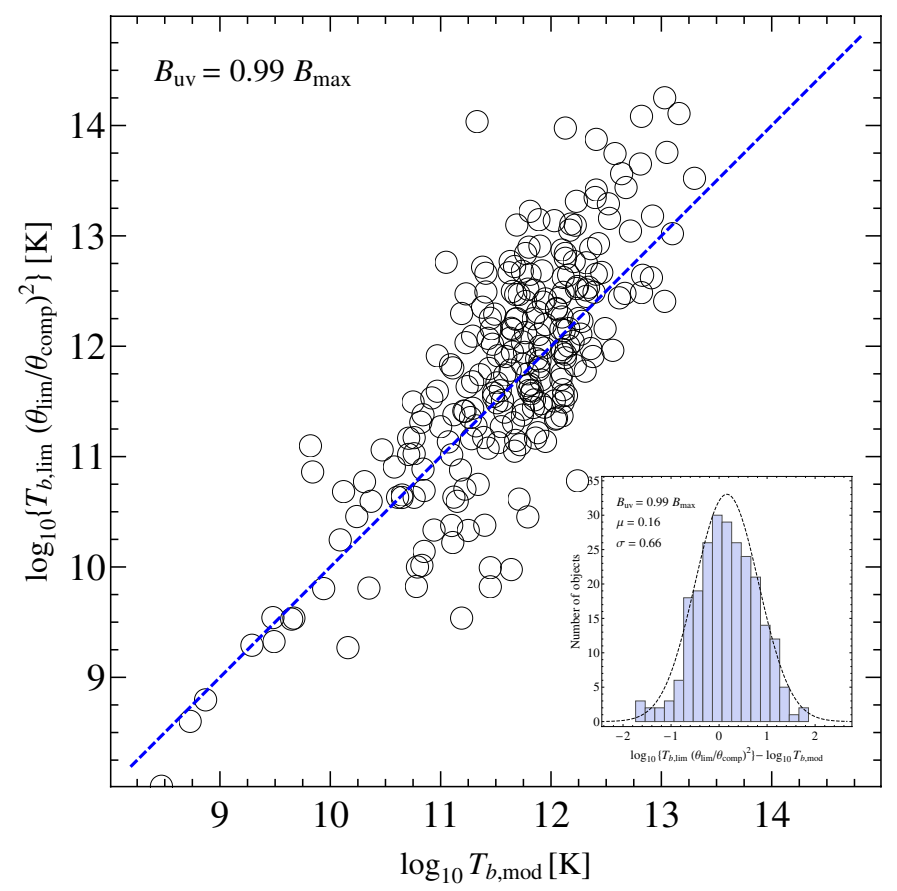

Fig. 8. Correction of the distribution shown in Fig. 6 for the square of the resolution factor $\theta_{\text {lim }} / \theta_{\text {comp }}$. The corrected $T_{\mathrm{b}, \mathrm{lim}}$ distribution is statistically very close to the $T_{\mathrm{b} \text {,mod }}$ obtained from the model-fitting analysis. The values of $T_{\mathrm{b} \text {,lim }}$ are on average $40 \%$ higher than the respective values of $T_{\mathrm{b}, \bmod }$. This is also demonstrated by the residual logarithmic distribution of the $T_{\mathrm{b}, \lim } / T_{\mathrm{b}, \bmod }$ ratio (inset), which can be approximated by the Gaussian PDF with $\mu=0.16$ and $\sigma=0.66$.

that the highest brightness is realized in a region that is smaller than the major axis of the Gaussian component describing the core region. Again, this would be the case for a quasi-stationary conical jet or a jet dominated by the instability patterns. In each of these situations, the observed jet brightness would be largely determined by its transverse dimension. If this is the case, $T_{\mathrm{b}, \mathrm{lim}}$ could be reconciled with $T_{\mathrm{b} \text {,mod }}$ derived under the assumption that only the minor axis, $\theta_{\min }$, of the Gaussian component is relevant for determining the jet brightness.

This hypothesis is tested in Fig. 7 by comparing $T_{\mathrm{b}, \lim }$ to $T_{\mathrm{b} \text {,mod }} \theta_{\text {maj }} / \theta_{\text {min }}$, which has the effect of calculating the size of the core region as $\pi \theta_{\min }^{2} / 4$. This simple correction brings the two estimates to a very good agreement, as demonstrated by both the average ratio between the two estimates and the distribution of logarithmic residuals of this ratio. The only notable discrepancy between the two estimates is the persistently higher $T_{\mathrm{b}, \lim }$ values observed for the low $T_{\mathrm{b} \text {,mod }} \lesssim 3 \times 10^{10}$. Such brightness temperatures are typically measured in objects in which the cores are strongly resolved along both axes of the fitted Gaussians, hence they may require a correction for the resolution along the minor axis of the Gaussian as well.

Such a correction can be performed by substituting the fitted effective size, $\theta_{\text {comp }}=\sqrt{\theta_{\min } \theta_{\text {maj }}}$ of the Gaussian component with its respective resolution limit $\theta_{\text {lim }}$ derived in Kovalev et al. (2005). This operation should account for resolving the core region along and across the jet direction as marked by the position angle of the major axis of the fitted Gaussian component. Applying this correction is illustrated by Fig. 8, which shows an excellent agreement between the two estimates of the brightness temperature and indeed effectively brings the low $T_{\mathrm{b} \text {,mod }}$ outliers to the main correlation trend. 


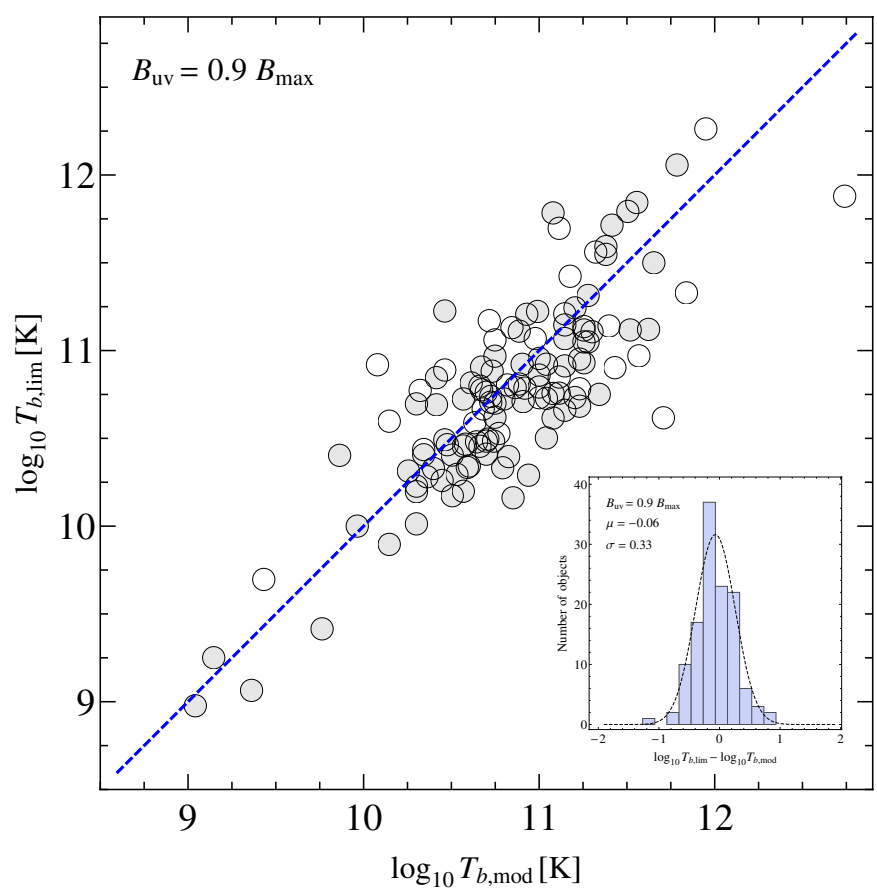

Fig. 9. Comparison of $T_{\mathrm{b}, \mathrm{lim}}$ (circular Gaussian approximation) and $T_{\mathrm{b}, \text { mod }}$ estimates obtained from the $86 \mathrm{GHz}$ survey data (Lee et al. 2008). Open circles indicate objects with lower limits on $T_{\mathrm{b}, \text { mod }}$. The dashed line marks the one-to-one correspondence between the two estimates. For each object, the $T_{\mathrm{b}, \mathrm{im}}$ is estimated from the $86 \mathrm{GHz}$ data at $R_{\mathrm{uv}} \geq 0.90 B_{\max }$ to restrict the visibility information to the most compact structures. The resulting $T_{\mathrm{b}, \mathrm{im}}$ are essentially equal to $T_{\mathrm{b}, \text { mod }}$. The residual logarithmic distribution of the $T_{\mathrm{b}, \mathrm{lim}} / T_{\mathrm{b} \text {,mod }}$ ratio (inset) can be approximated by the Gaussian PDF with $\mu=-0.06$ and $\sigma=0.33$.

The examples set by substituting the measured core size by either $\theta_{\min }$ or $\theta_{\text {lim }}$ indicate that the visibility-based estimates of $T_{\mathrm{b}, \mathrm{lim}}$ can be related to brightness temperature estimates obtained from Gaussian model fitting and that the $T_{\mathrm{b}, \text { lim }}$ estimates present a reliable account of brightness temperatures measured at the limit of angular resolution of the respective interferometric data sets.

\subsection{Results from the CMVA survey data}

If $T_{\mathrm{b}, \mathrm{lim}}$ is indeed determined by the limiting resolution of the data, the difference between the $T_{\mathrm{b}, \lim }$ and $T_{\mathrm{b} \text {,mod }}$ estimates should become progressively smaller with increasing $q_{\max }=$ $B_{\max } / \lambda_{\text {obs }}$ and for model fitting performed with circular Gaussian components (essentially forcing model fitting to be more responsive to the resolution limit). Both these conditions are fulfilled with the $86 \mathrm{GHz}$ data from the CMVA survey.

An analysis of the $86 \mathrm{GHz}$ survey data presented in Fig. 9 demonstrates that at spatial frequencies $q \geq 2 \mathrm{G} \lambda$, the estimates $T_{\mathrm{b}, \mathrm{lim}}$ and $T_{\mathrm{b} \text {,mod }}$ yield similar brightness temperatures. This indicates that at these spatial frequencies, the visibility distribution is strongly dominated by the most compact structure, which is reflected both in the modelfit results and in the visibility-based estimates of $T_{\mathrm{b}, \mathrm{lim}}$. It can therefore be expected that the visibilitybased estimates provide reliable brightness temperature limits for RadioAstron space VLBI measurements (Kardashev et al. 2013) and millimeter VLBI observations (Doeleman et al. 2012), reaching frequency spacings well in excess of $1 \mathrm{G} \lambda$.

\subsection{Comparisons with $T_{\mathrm{b}, \min }$}

Statistical comparisons of $T_{\mathrm{b} \text {,mod }}$ and $T_{\mathrm{b}, \mathrm{lim}}$ with the minimum brightness temperature $T_{\mathrm{b} \text {,min }}$ can also be made, although the latter estimate may be affected by a general flux density bias in the surveys. We recall that $T_{\mathrm{b}, \mathrm{min}}$ only depends on the absolute value of the visibility amplitude, while $T_{\mathrm{b}, \mathrm{lim}}$ effectively represents the signal-to-noise ratio (SNR) of the visibility.

The average ratios of $T_{\mathrm{b}, \lim } / T_{\mathrm{b} \text {, mod }}$ are $2.0_{-1.1}^{+2.3}$ and $1.6_{-0.5}^{+0.7}$ for the MOJAVE and CMVA samples, respectively. These values are similar to the ratios of 2.6 and 2.1 obtained in Sect. 2 from the full-track VLBI data on 3C 345 and NGC 1052. The rather large spread of these average ratios is indicative of random errors in the flux density scales of both surveys (which can result from self-calibration applied during imaging the survey data). The lower bounds of both averages are $\approx 1$, which can either reflect a relatively low SNR of the long-baseline visibilities or suggest that a systematic bias may also affect the flux density scales.

The average $T_{\mathrm{b}, \mathrm{lim}} / T_{\mathrm{b} \text {,mod }}$ ratios imply $S N R_{\mathrm{MOJAVE}}=3.9$ and $S N R_{\mathrm{CMVA}}=2.8$ on the baselines at which the brightness temperature limits are estimated. This is indeed similar to the measured SNR on the longest baselines in the survey data.

The average SNR can also be used to calculate the amount of bias in the flux density scale that is needed to bring the survey results closer to the results from the two full-track cases considered. In both cases, a positive flux density bias of about $20 \%$ on the longest baselines would reconcile the survey averages with those of the full-track data. Hence, this can also be a viable possibility, in particular for the CMVA data that have been collected in three long observing runs.

The comparison of $T_{\mathrm{b} \text {,min }}$ and $T_{\mathrm{b} \text {,mod }}$ obtained from the MOJAVE data yields an average ratio $T_{\mathrm{b}, \min } / T_{\mathrm{b}, \bmod }=2.3$, while a ratio of $\leq 1$ is expected. This discrepancy probably results from the same potential problems as discussed in Sect. 3.2 in connection with using elliptical modelfits to describe the core region. Indeed, applying either the $\theta_{\min }$ or the $\theta_{\text {lim }}$ correction described in Sect. 3.2 reduces this ratio to 0.7. This lends further support to the conclusion that elliptical modelfits may not be optimal for estimating the brightness temperature in compact jets because it is effectively determined by the transverse dimension of the flow. Following this line of argument, the CMVA data (fitted by circular Gaussian and providing substantially longer baselines for estimating $T_{\mathrm{b}, \min }$ ) should yield a better value of the average $T_{\mathrm{b}, \min } / T_{\mathrm{b} \text {,mod }}$ ratio. This is indeed the case, with the average $T_{\mathrm{b}, \min } / T_{\mathrm{b}, \text { mod }}=0.5$ measured from the CMVA data.

\subsection{General implications for $T_{\mathrm{b}}$ measurements}

Overall, the comparisons of $T_{\mathrm{b}, \mathrm{lim}}$ and $T_{\mathrm{b} \text {,mod }}$ made using the MOJAVE and the CMVA survey data indicate that brightness temperature estimates obtained from the visibility flux density at longest baselines (highest spatial frequencies) provide suitable limits on the brightness temperature of the most compact emitting regions in radio sources. These comparisons also suggest that visibility-based estimates may even be more realistic in case of complex structure of the emitting region (i.e., a marginally transversely resolved flow, such as a compact conical jet, or a flow with thread-like patterns embedded).

The same situation may be realized for other types of objects studied with interferometric measurements, for instance, in radio interferometry observations of young supernovae (Marcaide et al. 2009), radio (Dyck et al. 1998) and optical (Cusano et al. 2012; Ohnaka et al. 2013; Arroyo-Torres et al. 2014) interferometry studies of resolved stars, and optical interferometry 
observations of protoplanetary disks (Malbet et al. 2005), recurrent novae (Chesneau et al. 2007), and active galaxies (Weigelt et al. 2012). In optical interferometry experiments that measure the normalized visibility spectrum $V_{q} / V_{0}$, the zero-spacing flux, $V_{0}$, may need to be determined in a separate measurement (cf. Cusano et al. 2012). In all of these cases, brightness temperature estimates can be made with a visibility response calculated for a specific pattern of brightness distribution (see Appendix A). For objects with a complex internal structure, this would correspond to estimating a pattern-averaged brightness temperature, while the brightness temperature of the most compact regions can still be estimated by using the data from the longest baselines and applying the generic Gaussian case described in Sect. 2.

The arguments presented above demonstrate that, for a given interferometric measurement, a range of plausible brightness temperatures of the most compact emitting structure can be determined from the visibility amplitude and its error measured at the longest baselines contributing to the measurement. This procedure effectively addresses the brightness temperature of emitting regions detected at the limiting resolution of the measurement.

In summary, our analysis leads to the following basic conclusions about applying visibility data to constrain the brightness temperature in astrophysical objects:

1. A measurement of visibility amplitude, $V_{q}$, alone is sufficient to constrain the minimum brightness temperature, $T_{\mathrm{b}, \min }$, that can be derived from this visibility under the assumption that the brightness distribution of the target object can be described by a Gaussian function. This holds for both circular and elliptical Gaussians and indeed for any visibility distribution that has an inflection point (for instance, for any visibility distribution that can be approximated by a Bessel function of the first kind).

2. If the brightness temperature of an emitting region with a putative Gaussian brightness distribution is constrained using an assumed limit on the zero-spacing flux density, $V_{0}$, the nature of the resulting constraint depends on the ratio $V_{0} / V_{q}$. For $V_{0}>e V_{q}$, lower/upper limits on $V_{0}$ provide lower/upper limits on $T_{\mathrm{b}}$. For $V_{0}<e V_{q}$, lower/upper limits on $V_{0}$ provide upper/lower limits on $T_{\mathrm{b}}$.

3. A measurement of visibility amplitude, $V_{q}$, and its rms error, $\sigma_{q}$, can be used to calculate the maximum brightness temperature, $T_{b \text {,lim }}$ that can be obtained under assumption that the emitting region is marginally resolved.

4. At spatial frequencies higher than $\sim 200 \mathrm{M} \lambda$, the interval $\left[T_{\mathrm{b}, \min }, T_{\mathrm{b}, \mathrm{lim}}\right]$ provides a reliable bracketing for the brightness temperature of the most compact structure in the target object.

5. Applying the analysis to samples of radio sources with brightness temperatures derived from model fitting the compact structure indicates that the brightness temperature in the relativistic jets may be largely determined by the transverse dimension of the flow.

6. The visibility-based estimates of brightness temperature can offer a suitable tool for constraining this physical parameter in a wide range of experiments in which a full reconstruction of the source structure is not feasible.

Acknowledgements. This research has made use of data from the MOJAVE database that is maintained by the MOJAVE team (Lister et al. 2009, AJ, 137,
3718) and of data obtained with the Global Millimeter VLBI Array, which consists of telescopes operated by the MPIfR, IRAM, Onsala, Metsahovi, Yebes and the VLBA. The VLBA is an instrument of the National Radio Astronomy Observatory, a facility of the National Science Foundation operated under cooperative agreement by Associated Universities, Inc.

\section{Appendix A: Visibility limits on the brightness temperature derived for specific patterns of brightness distribution}

Visibility limits on brightness temperature can be obtained by combining the expression for brightness temperature,

$$
\begin{aligned}
T_{\mathrm{b}} & =\frac{h v}{k} \ln ^{-1}\left(1+\frac{2 h v^{3}}{I(r) c^{2}}\right)=\frac{h v}{k} \ln ^{-1}\left(1+\mathcal{I}_{\mathrm{b}}\right) \\
& \rightarrow \frac{I(r) c^{2}}{2 k v^{2}} \quad \text { for } h v \ll k T,
\end{aligned}
$$

with the visibility function, $V$, calculated for a specific, circularly symmetric pattern of brightness distribution, $I(r)$, observed with an interferometer at a spatial harmonic, $q$, which yields a visibility measurement $V(q)$ and its rms error $\sigma(q)$. Here $h$ is the Planck constant, $k$ is the Boltzmann constant, $c$ is the light speed, and $v$ is the frequency of measurement.

For the Planck regime, the term $\mathcal{I}_{r}$ is derived for each pattern. Full expressions for $T_{\mathrm{b}}$ are given for the Rayleigh-Jeans regime. The general form of brightness distribution pattern is chosen to be $I(r)=\eta_{\mathrm{d}} S_{\text {tot }} f(r)$, where $S_{\text {tot }} \equiv V(0)$ is the total or zero-spacing intensity of the pattern, and $f(r)$ and $\eta_{\mathrm{d}}$ are the respective pattern-specific radial dependence of brightness and its normalization factor (generally dependent on the size $d$ of the pattern). The normalization, $\eta_{\mathrm{d}}$, is chosen so that it provides a Fourier transform $\mathcal{F} I(r)$ of the form $V(q)=V(0) \mathcal{F} f(r)$. To simplify the expressions derived, $I_{r}, V_{0}$ and $\left(V_{q}, \sigma_{q}\right)$ denote $I(r)$, $V(0)$ and $(V(q), \sigma(q))$ in the discussion below.

With the adopted normalization, a generic solution for the size $d_{q}$ of the pattern can be determined by solving $\mathcal{F} I_{r}=$ $V_{q} /\left(V_{0}\right)$ for $d$. The minimum brightness temperature supported by the visibility measurement $V_{q}$ is obtained by substituting $I_{r}$ with $I_{r}\left(d \rightarrow d_{q}\right)$ in Eq. (A.1) and finding the value of $V_{0}$ that minimizes the respective $T_{\mathrm{b}}$ given by this equation (e.g., deriving $V_{0}$ from the condition $\mathrm{d} T_{\mathrm{b}} / \mathrm{d} V_{0}=0$ ).

To derive the maximum measurable brightness temperature, the characteristic size, $d_{\text {lim }}$, of the pattern is obtained from the relation $\mathcal{F} I_{r}=V_{q} /\left(V_{q}+\sigma_{q}\right)$. The maximum measurable brightness temperature $T_{\mathrm{b}, \mathrm{lim}}$ is then calculated by substituting $I_{r}$ in Eq. (A.1) with $I_{r}\left(d \rightarrow d_{\text {lim }}\right)$.

Results of the calculations are presented in Table A.1 for a circular Gaussian component, a disk of uniform brightness, and a spherical shell of finite thickness. The characteristic size $d$ represents, respectively, the full width at half maximum of the Gaussian component, the diameter of the disk, and the outer diameter of the shell. The spherical shell is further defined by its thickness $\delta_{r}=(1-\alpha) d / 2$ (with $\left.0 \leq \alpha \leq 1\right)$. Thus, $\alpha=0$ describes an infinitely thin shell and $\alpha \rightarrow 1$ describes a filled, optically thin sphere. Calculations for the spherical shell are made separately for the average shell brightness and for the peak brightness $I_{r \text {,peak }}$ in the shell, realized at $r=\alpha d / 2$, with the resulting $I_{r, \text { peak }}=6 S_{\text {tot }} / \pi d^{2}\left(1-\alpha^{3}\right)^{1 / 2}$. 
A. Lobanov: Brightness temperature from interferometric visibilities

Table A.1. Visibility brightness temperature estimates for specific patterns of brightness distribution.

\begin{tabular}{|c|c|c|c|c|}
\hline & \multirow{2}{*}{$\begin{array}{c}\text { Circular Gaussian } \\
\text { component }\end{array}$} & \multirow{2}{*}{$\begin{array}{l}\text { Uniformly bright } \\
\text { disk }\end{array}$} & \multicolumn{2}{|c|}{ Optically thin shell of finite thickness } \\
\hline & & & shell average & peak brightness \\
\hline$I_{r}$ & $\frac{2 \sqrt{\ln 2}}{\sqrt{\pi}} \frac{S_{\text {tot }}}{d} \exp \left(-\frac{4 \ln 2 r^{2}}{d^{2}}\right)$ & $S_{\text {tot }} \begin{cases}4 /\left(\pi d^{2}\right), & r \leq d / 2, \\
0 & r>d / 2\end{cases}$ & 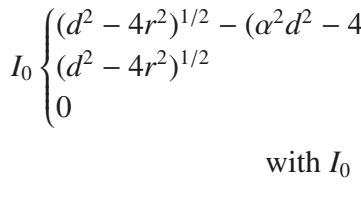 & $\begin{array}{l})^{1 / 2} \quad r<\alpha d \\
\alpha d \leq 2 r \leq d, \\
2 r>d \\
\frac{6 S_{\text {tot }}}{\pi d^{3}\left(1-\alpha^{3}\right)}\end{array}$ \\
\hline$T_{\mathrm{b}}$ & $\frac{2 \ln 2}{\pi k} \frac{S_{\mathrm{tot}} \lambda^{2}}{d^{2}}$ & $\frac{2}{\pi k} \frac{S_{\mathrm{tot}} \lambda^{2}}{d^{2}}$ & $\frac{2}{\pi k} \frac{S_{\mathrm{tot}} \lambda^{2}}{d^{2}}$ & $\frac{3}{\pi k} \frac{S_{\mathrm{tot}} \lambda^{2}}{d^{2}\left(1-\alpha^{3}\right)^{1 / 2}}$ \\
\hline $\mathcal{I}_{\mathrm{b}}$ & $\frac{\pi h v}{2 \ln 2} \frac{d^{2}}{S_{\text {tot }} \lambda^{2}}$ & $\frac{\pi h v}{2} \frac{d^{2}}{S_{\text {tot }} \lambda^{2}}$ & $\frac{\pi h v}{2} \frac{d^{2}}{S_{\text {tot }} \lambda^{2}}$ & $\frac{\pi h v}{3} \frac{d^{2}\left(1-\alpha^{3}\right)^{1 / 2}}{S_{\text {tot }} \lambda^{2}}$ \\
\hline$V_{q}$ & $V_{0} \exp \left(-\frac{\pi^{2} q^{2} d^{2}}{4 \ln 2}\right)$ & $\begin{array}{l}V_{0} \frac{2 J_{1}(\pi d q)}{\pi d q} \approx \\
V_{0}\left(1-\frac{\pi^{2} d^{2} q^{2}}{8}\right)\end{array}$ & $\begin{array}{r}V_{0} \frac{3}{\left(1-\alpha^{3}\right) \pi^{3} d^{3} q^{3}}[\sin (\pi d q \\
\\
\approx V_{0}(1-\end{array}$ & $\begin{array}{l}\pi d q \cos (\pi d q) \\
\sin (\alpha \pi d q)+\alpha \pi d q \cos (\alpha \pi d q)] \\
\left.\frac{\pi^{2} d^{2} q^{2}}{10} \frac{1-\alpha^{5}}{1-\alpha^{3}}\right)\end{array}$ \\
\hline$d_{q}$ & $\frac{2 \sqrt{\ln 2}}{\pi} \frac{1}{q} \sqrt{\ln \left(V_{0} / V_{q}\right)}$ & $\frac{2 \sqrt{2}}{\pi q} \sqrt{1-V_{q} / V_{0}}$ & $\frac{\sqrt{10}}{\pi q}\left(\frac{1-}{1-}\right.$ & )$^{1 / 2} \sqrt{1-V_{q} / V_{0}}$ \\
\hline$T_{\mathrm{b}, \mathrm{q}}$ & $\frac{\pi}{2 k} \frac{B^{2} V_{0}}{\ln \left(V_{0} / V_{q}\right)}$ & $\frac{\pi}{4 k} \frac{B^{2} V_{0}^{2}}{V_{0}-V_{q}}$ & $\frac{\pi}{5 k} \frac{1-\alpha^{5}}{1-\alpha^{3}} \frac{B^{2} V_{0}^{2}}{V_{0}-V_{q}}$ & $\frac{3 \pi}{10 k} \frac{1-\alpha^{5}}{\left(1-\alpha^{3}\right)^{3 / 2}} \frac{B^{2} V_{0}^{2}}{V_{0}-V_{q}}$ \\
\hline $\mathcal{I}_{\mathrm{b}, \mathrm{q}}$ & $\frac{2 h v}{\pi} \frac{\ln \left(V_{0} / V_{q}\right)}{B^{2} V_{0}}$ & $\frac{4 h v}{\pi} \frac{V_{0}-V_{q}}{B^{2} V_{0}^{2}}$ & $\frac{5 h v}{\pi} \frac{1-\alpha^{3}}{1-\alpha^{5}} \frac{V_{0}-V_{q}}{B^{2} V_{0}^{2}}$ & $\frac{10 h v}{3 \pi} \frac{\left(1-\alpha^{3}\right)^{3 / 2}}{1-\alpha^{5}} \frac{V_{0}-V_{q}}{B^{2} V_{0}^{2}}$ \\
\hline$T_{\mathrm{b}, \min }$ & $\frac{\pi e}{2 k} B^{2} V_{q}$ & $\frac{\pi}{k} B^{2} V_{q}$ & $\frac{4 \pi}{5 k} \frac{1-\alpha^{5}}{1-\alpha^{3}} B^{2} V_{q}$ & $\frac{6 \pi}{5 k} \frac{1-\alpha^{5}}{\left(1-\alpha^{3}\right)^{1 / 2}} B^{2} V_{q}$ \\
\hline $\mathcal{I}_{\mathrm{b}, \min }$ & $\frac{2 h v}{\pi e B^{2} V_{q}}$ & $\frac{h v}{\pi B^{2} V_{q}}$ & $\frac{5 h v}{4 \pi} \frac{1-\alpha^{3}}{1-\alpha^{5}} \frac{1}{B^{2} V_{q}}$ & $\frac{5 h v}{6 \pi} \frac{\left(1-\alpha^{3}\right)^{3 / 2}}{1-\alpha^{5}} \frac{1}{B^{2} V_{q}}$ \\
\hline$T_{\mathrm{b}, \mathrm{lim}}$ & $\frac{\pi}{2 k} \frac{B^{2}\left(V_{q}+\sigma_{q}\right)}{\ln \left[\left(V_{q}+\sigma_{q}\right) / V_{q}\right]}$ & $\frac{\pi}{4 k} \frac{B^{2}\left(V_{q}+\sigma_{q}\right)^{2}}{\sigma_{q}}$ & $\frac{\pi}{5 k} \frac{1-\alpha^{5}}{1-\alpha^{3}} \frac{B^{2}\left(V_{q}+\sigma_{q}\right)^{2}}{\sigma_{q}}$ & $\frac{3 \pi}{10 k} \frac{1-\alpha^{5}}{\left(1-\alpha^{3}\right)^{3 / 2}} \frac{B^{2}\left(V_{q}+\sigma_{q}\right)^{2}}{\sigma_{q}}$ \\
\hline $\mathcal{I}_{\mathrm{b}, \mathrm{lim}}$ & $\frac{2 h v}{\pi} \frac{\ln \left[\left(V_{q}+\sigma_{q}\right) / V_{q}\right]}{B^{2}\left(V_{q}+\sigma_{q}\right)}$ & $\frac{4 h v}{\pi} \frac{\sigma_{q}}{B^{2}\left(V_{q}+\sigma_{q}\right)^{2}}$ & $\frac{5 h v}{\pi} \frac{1-\alpha^{3}}{1-\alpha^{5}} \frac{\sigma_{q}}{B^{2}\left(V_{q}+\sigma_{q}\right)^{2}}$ & $\frac{10 h v}{3 \pi} \frac{\left(1-\alpha^{3}\right)^{3 / 2}}{1-\alpha^{5}} \frac{\sigma_{q}}{B^{2}\left(V_{q}+\sigma_{q}\right)^{2}}$ \\
\hline
\end{tabular}

Notes. $I_{r}$ - brightness distribution function; $T_{\mathrm{b}}$ - brightness temperature estimated from $I_{r} ; V_{q}$ - visibility function; $d_{q}$ - pattern size, estimated from the visibility ratio $V_{q} / V_{0} ; T_{\mathrm{b}, \mathrm{q}}$ - brightness temperature corresponding to $d_{q} ; T_{\mathrm{b}, \min }$ - minimum brightness temperature, obtained with $V_{0}=e V_{q}$ for the Gaussian component and $V_{0}=2 V_{q}$ for the disk and the shell; $T_{\mathrm{b}, \text { lim }}-$ limiting brightness temperature, obtained with $V_{0}=V_{q}+\sigma_{q}$. For the brightness temperature estimates, the respective $\mathcal{I}$ expression give the $\mathcal{I}_{\mathrm{b}}$ term in the Planck form of the brightness temperature equation.

\section{References}

Arroyo-Torres, B., Martí-Vidal, I., Marcaide, J. M., et al. 2014, A\&A, 566, A88 Berger, J.-P. 2003, in EAS Pub. Ser. 6, eds. G. Perrin, \& F. Malbet, 23

Blandford, R. D., \& Königl, A. 1979, ApJ, 232, 34

Chesneau, O., Nardetto, N., Millour, F., et al. 2007, A\&A, 464, 119

Cusano, F., Paladini, C., Richichi, A., et al. 2012, A\&A, 539, A58

Doeleman, S. S., Fish, V. L., Schenck, D. E., et al. 2012, Science, 338, 355

Dyck, H. M., van Belle, G. T., \& Thompson, R. R. 1998, AJ, 116, 981

Homan, D. C., Kovalev, Y. Y., Lister, M. L., et al. 2006, ApJ, 642, L115

Horiuchi, S., Fomalont, E. B., Taylor, W. K., et al. 2004, ApJ, 616, 110

Kardashev, N. S., Khartov, V. V., Abramov, V. V., et al. 2013, Astron. Rep., 57,
Kellermann, K. I., Lister, M. L., Homan, D. C., et al. 2004, ApJ, 609, 539 Kovalev, Y. Y., Kellermann, K. I., Lister, M. L., et al. 2005, AJ, 130, 2473 Lee, S.-S., Lobanov, A. P., Krichbaum, T. P., et al. 2008, AJ, 136, 159 Lee, S.-S., Han, M., Kang, S., et al. 2013, in EPJ Web Conf., 61, 7007 Lobanov, A. P., \& Zensus, J. A. 2001, Science, 294, 128

Lobanov, A. P., Krichbaum, T. P., Graham, D. A., et al. 2000, A\&A, 364, 391

Malbet, F., Lachaume, R., Berger, J.-P., et al. 2005, A\&A, 437, 627

Marcaide, J. M., Martí-Vidal, I., Alberdi, A., et al. 2009, A\&A, 505, 927

Ohnaka, K., Hofmann, K.-H., Schertl, D., et al. 2013, A\&A, 555, A24

Petrov, L., Lee, S.-S., Kim, J., et al. 2012, AJ, 144, 150

Weigelt, G., Hofmann, K.-H., Kishimoto, M., et al. 2012, A\&A, 541, L9 